Pathologe 2019 40 (Suppl 3):S409-S410 https://doi.org/10.1007/s00292-019-00659-w Online publiziert: 11. November 2019

(c) Springer Medizin Verlag $\mathrm{GmbH}$, ein Teil von Springer Nature 2019

\section{F. Fend}

Abteilung Allgemeine und Molekulare Pathologie und Pathologische Anatomie, Institut für Pathologie und Neuropathologie, Universitätsklinikum Tübingen, Eberhard-Karls-Universität Tübingen, Tübingen, Deutschland

\title{
Hermine Valeria Gärtner
}

\author{
14.08.1942-20.07.2018
}

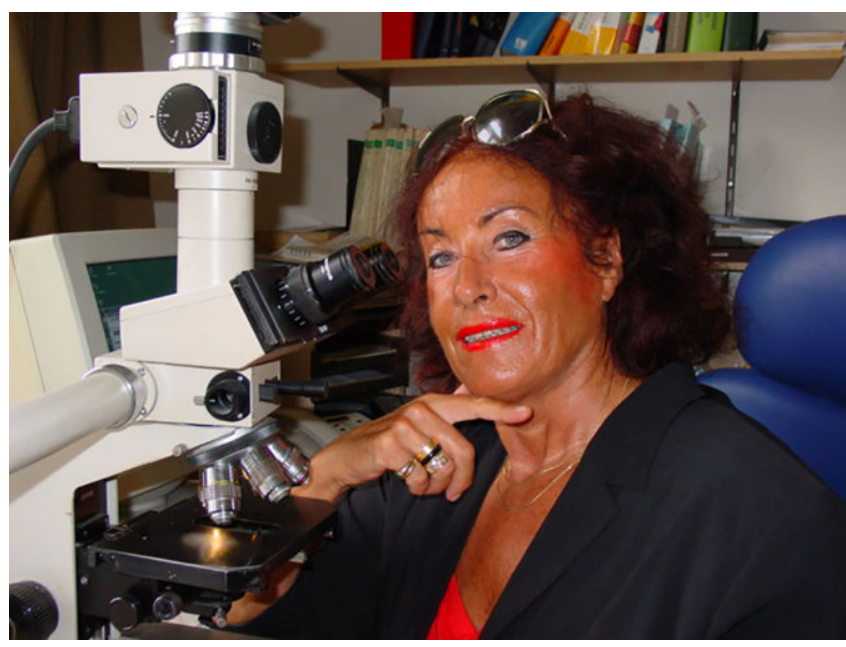

Hermine Valeria Gärtner

Frau Prof. Dr. Hermine Valeria Gärtner, Mitglied unserer Gesellschaft, ist am 20.07.2018 in Tübingen verstorben. Valeria Gärtner wurde am 14.08.1942 in Welzow in der Niederlausitz geboren. Nach dem Krieg musste sie mit ihrer Familie fliehen und fand schließlich Unterkunft bei ihrer Tante in Esslingen. Aufgrund ihrer Begabung wechselte sie trotz ihres Status als armes Flüchtlingskind bald von der Mittelschule ins Gymnasium. Im Wintersemester 1961/62 begann sie das Medizinstudium in Tübingen, musste aber zur Finanzierung des Studiums immer wieder arbeiten, u.a. hinter der Bar eines bekannten Akademikertreffs in Tübingen. Dort und in Vorlesungen des Studium Generale bei Geistesgrößen wie Paul Thieme und Walter Jens wurde wohl die Grundlage für ihr zeitlebens anhaltendes Interesse an den Geisteswissenschaften gelegt. Nach dem Physikum absolvierte sie Gastsemester in München, Kiel und Wien. Auf ihrer letzten Station hörte sie noch Vorlesungen bei dem großen $\mathrm{Pa}$ thologen Hermann Chiari. Initial galt ihr
Interesse der Psychiatrie, doch nach dem Tod ihres Doktorvaters Walter Schulte wandte sie sich von der Psychiatrie ab. Nach Absolvierung der Medizinalpraktikantenzeit am Krankenhaus München Bogenhausen wurde ihr eine Stelle am Institut für Pathologie Tübingen angeboten. Beim Vorstellungsgespräch antwortete sie auf die Frage von Adalbert Bohle, was sie in der Pathologie machen wolle, mit: „Ich will mich habilitieren!“, ein für die damalige Zeit durchaus ungewöhnliches Ansinnen für eine Frau in der Pathologie. Unter ihrem Mentor Prof. Bohle, dessen Institut ganz im Zeichen der Niere stand, entwickelte sich Valeria Gärtner rasch zu einer hervorragenden Nephropathologin; ein zweites Standbein war die Hämatopathologie. Im Sommersemester 1978 habilitierte sie sich mit dem Titel „Perimembranöse Glomerulonephritis. Prototyp einer Immunkomplexerkrankung“. Auf den Jahrestagungen der Deutschen Gesellschaft für $\mathrm{Pa}$ thologie war sie anfangs oft die einzige weibliche Vortragende. Neben vielen in- ternationalen Kongressen waren die von Walter Schulz veranstalteten Bamberger Nephrologischen Seminare ein Fixpunkt ihrer Vortragstätigkeit. Der Schwerpunkt ihrer wissenschaftlichen Tätigkeit lag auf dem Gebiet der Nephropathologie. Als Themen ihrer Arbeiten sind die membranoproliferative Glomerulonephritis, die präeklamptische Nephropathie, die IgANephritis und die Amyloidose zu nennen. Eine Besonderheit stellte ein Fallbericht im New England Journal of Medicine dar, in dem sie die akzidentelle Transplantation eines Sarkoms in die Hand eines Chirurgen durch eine intraoperativ erlittene Stichverletzung beschrieb und genetisch nachweisen konnte, dass der Tumor vom operierten Patienten stammte.

Neben ihrer wissenschaftlichen und diagnostischen Tätigkeit widmete sich Valeria Gärtner in zunehmendem Maße der Arbeit in Kollegialorganen der medizinischen Fakultät. Im Jahr 1992 wurde sie zur Frauenbeauftragten, später Gleichstellungsbeauftragten ernannt; eine Tätigkeit, die sie bis zum Jahr 2012 mit viel Engagement und Herzblut ausübte und in der sie sich nicht scheute, mit markanter Rhetorik und prägnantem Auftreten ihre Ansichten gegen Widerstände $\mathrm{zu}$ verteidigen.

Neben der Pathologie und dem Engagement an der medizinischen Fakultät hatte Valeria Gärtner eine Vielzahl von Interessen, und sie nahm regen Anteil am kulturellen und geistigen Leben in Tübingen. Reisen und das Kennenlernen fremder Kulturen war neben der Pathologie ihre große Leidenschaft. Tibet wurde zu ihrer Herzensheimat, in die sie insgesamt 17 Reisen führten. Als begeisterte Fotografin stellte sie eine Ausstellung über Tibet zusammen, die in ganz Deutsch- 


\section{Nachrufe}

land in vielen Krankenhäusern gezeigt wurde.

Valeria Gärtner war nicht nur eine der ersten Frauen, die in der Deutschen Pathologie Karriere machte und eine Kämpferin für die Rolle der Frauen in der Medizin, sondern auch eine bemerkenswerte Persönlichkeit, die jedem, der sie Kennenlernen durfte, in Erinnerung bleiben wird. Passend $\mathrm{zu}$ ihrem ereignisreichen und manchmal turbulenten, sicher nie langweiligen Leben verfasste sie 2015 eine lesenswerte Autobiografie „Mein Leben mit der Maske - durch Leidenschaft in die Sphären der Macht", in der die akademische Pathologie in Deutschland, die universitäre Medizin und die Medizinische Fakultät Tübingen aus manchmal ungewohntem Blickwinkel, gelegentlich auch durchaus provokant, aber nie langweilig dargestellt wird. Für mich persönlich war sie immer eine interessante und unterhaltsame Gesprächspartnerin, die bis zuletzt großen Anteil an den Entwicklungen am Institut und der Fakultät nahm. Mit Valeria Gärtner verliert unsere Gesellschaft eine prägnante Vertreterin des Fachs und eine Vorkämpferin für die Frauen in der Pathologie.

\section{Falko Fend}

Tübingen

\section{Korrespondenzadresse}

\section{Prof. Dr. F. Fend}

Abteilung Allgemeine und Molekulare Pathologie und Pathologische Anatomie, Institut für Pathologie und Neuropathologie, Universitätsklinikum Tübingen, Eberhard-KarlsUniversität Tübingen

Liebermeisterstraße 8, 72076 Tübingen,

Deutschland

falko.fend@med.uni-tuebingen.de

The supplement containing this article is not sponsored by industry. 